\title{
DNA Binding Domain
}

National Cancer Institute

\section{Source}

National Cancer Institute. DNA Binding Domain. NCI Thesaurus. Code C13425.

A region of a protein that interacts with DNA. Among the various recog nized structural motifs present in DNA binding domains are the helix-loop-helix and the zinc-finger. 